\title{
Dedicated Filter for Robust Occupancy Grid Mapping
}

\author{
K.S. Nagla*, Moin Uddin**, Dilbag Singh* \\ ${ }^{*}$ Dr. BR Ambedkar National Institute of Technology Jalandhar, India-144011 \\ ${ }^{* *}$ Jamia Hamdard University New Delhi, India- 110062
}

\begin{tabular}{l}
\hline \hline Article Info \\
\hline Article history: \\
Received Jul 5, 2014 \\
Revised Nov 4, 2014 \\
Accepted Nov 25, 2014 \\
\hline
\end{tabular}

Keyword:

Bayesian inference

Robust occupancy grid

Sensor fusion

Sonar and Laser sensor

Mapping

\begin{abstract}
Sensor based perception of the environment is an emerging area of the mobile robot research where sensors play a pivotal role. For autonomous mobile robots, the fundamental requirement is the convergent of the range information in to high level internal representation. Internal representation in the form of occupancy grid is commonly used in autonomous mobile robots due to its various advantages. There are several sensors such as vision sensor, laser rage finder, and ultrasonic and infrared sensors etc. play roles in mapping. However the sensor information failure, sensor inaccuracies, noise, and slow response are the major causes of an error in the mapping. To improve the reliability of the mobile robot mapping multisensory data fusion is considered as an optimal solution. This paper presents a novel architecture of sensor fusion frame work in which a dedicated filter (DF) is proposed to increase the robustness of the occupancy grid for indoor environment. The technique has been experimentally verified for different indoor test environments. The proposed configuration shows improvement in the occupancy grid with the implementation of dedicated filters.
\end{abstract}

Copyright (C) 201x Institute of Advanced Engineering and Science. All rights reserved.

\section{Corresponding Author:}

KS Nagla

Department of ICE, Dr BR Ambedkar National Institute of Technology, Jalandhar-India

Email: naglaks@nitj.ac.in

Tel: +91-181-2690324, EPBAX: +91-181-2690301,+91-181-2690453 ext. 2911

Fax: $+91-181-2690320$

\section{INTRODUCTION}

Map formation in the form of occupancy grid has proven to be the powerful and efficient unifying solution for navigation and path planning in mobile robots. During navigation, the mobile robot receives complex data from different sensors and needs efficient processing to yield an optimal and consistent perception of the environment $[1,26,34,36$, and 37]. Mobile robot navigation is a combination of two fundamental components i.e. localization and mapping [3, 7, 10, 13, 20, and 35]. In literature it is considered as a complex problem, because for acquiring a map the robot needs good estimation of its localization and for localization the robot needs a consistent map [8]. Many researchers suggested techniques to represent the mobile robot mapping such as i.e. metric, topological and hybrid mapping approach [5, 6, 10, 12, 30, and 31]. Recently, hybrid methods of mapping and mobile cooperative network methods are explored for autonomous applications [24]. These approaches present complementary strengths and weaknesses [6, 8, and 32]. The occupancy grid is the metric approach considered as an efficient solution for mobile robot mapping in static environment and there has been most recent research applied to the dynamic situation also [37]. It represents a map of the environment as an evenly spaced field in the form of probability ranging from 0 to 1 . The accuracy of the grid mapping depends upon the clear detection of the boundaries of the obstacles in the environment hence the overall performance of the grid depends upon how accurately the generated grid is matching with real map of the environment. 
An accurate perception of the map in the form of occupancy grid enables the robot to complete complex tasks, accurately and reliably which depends upon the several factors such as kinematics and dynamic constraints of the mobile robot, sensing abilities, processing and control capabilities and more precisely it depends upon the complexity of the environment $[1,3]$. The range sensing accuracy is considered inherently difficult, as the small sensory noise introduced in the estimate of range may lead to the large distortion in the resultant map [5]. The noise reduction in the resultant grid is classified in to two basic processes of mapping i.e. pre-processing and post-processing of the information. This paper specifically focused upon the post-processing of the grid map.

The proposed technique is based upon analytical and mathematical analysis, in which the dedicated filters are implemented to the grid. Previously, for mapping with heterogeneous sensors, simple thresholding methods were used to eliminate local occlusions of occupancy grid [29]. In such situations a common thresholding for different sensors, causes decrease in the performance of the resultant grid. In the proposed method recursive Bayesian approach is used to update the map. The updated map is further filtered by using DF where, the optimal statistical values of the filter have been selected after quantitative and qualitative analysis of conventional mapping system. We compare the proposed technique with conventional method by using qualitative and quantitative analysis. The proposed technique is experimentally tested and validate for indoor office environment with a mobile robot equipped with sonar sensors and laser range finder. The robot is allowed move on a smooth surface in the environment consisting of various obstacles.

\section{ROBUST OCCUPANCY GRID MAPPING AND RELATED WORK}

Mobile robot mapping addresses the problem of acquiring physical environmental information around the robot with the help of sensors, where the sensor model converts the acquired information into an internal representation in the memory of the computer so that the controller may control the mobility of the robot accordingly. In practice the mobile robot has to deal with complex environmental problems, where the obstacles may be stationary or moving and the material properties of the obstacle may be highly absorbent or reflective (for acoustic and optical sensors). In past these factors have been handled carefully and put into practice to improve the accuracy of mapping by multisensory data fusion.

Multisensor data fusion is successfully implemented in mobile robots to improve the mapping. Literature shows several techniques such as weighted average method [21], Kalman filter [18], extended Kalman [19], Dempster-Shafer (DS) theory etc. is used. The performance value of occupancy grid is also improved by using logical sensors where online fusion algorithm and selection of most reliable sensor is taken up by with special rules of combination of fuzzy values [28]. Artificial Neural Networks used to map the environment in occupancy gird has been proven to be the robust and adaptive to environmental changes $[11,16,17,22,23$, and 25]. Post processing of the grid play crucial role to improve the occupancy grid mapping. Stepan et al. improved the robustness of occupancy grid by using (a) fusion of sensors with one occupancy grid (b) Fusion of sensor with two-occupancy grids (c) fusion of sensors with different precisions [29]. Stepan et al, elaborated to improve the robustness of grid by post processing using thresholding method and by combining an occupied cell from an accurate sensor such as vision sensor with a small neighborhood of corresponding cell from an inaccurate sensor such as sonar sensor. Stapen et al. (2005), in his research used a single threshold algorithm for different sensors that may reduce the performance of resultant grid in few cases [29]. In another post processing technique Jose-Luis Blanco et al. (2007) proposed to increase the robustness of the occupancy grid based on the extraction of feature descriptors by means of a polar coordinate transformation, around highly distinctive points. It presented a modest computation complexity and resulted robustness of grid even in the dynamic environment [15]. Author implemented an additional RANSAC-based method for estimating the map transformation using (SIFT) scale invariance feature transformation technique [2]. Most recent work of post processing of grid by using thesholding is presented by Boris Lau et al. (2012), [4].

\section{EXPERIMENTAL SETUP}

Mapping experiment conducted in the robotics laboratory by using four-wheeled mobile robot fitted with forward-looking sonar and laser range finder as shown in Figure 1. 


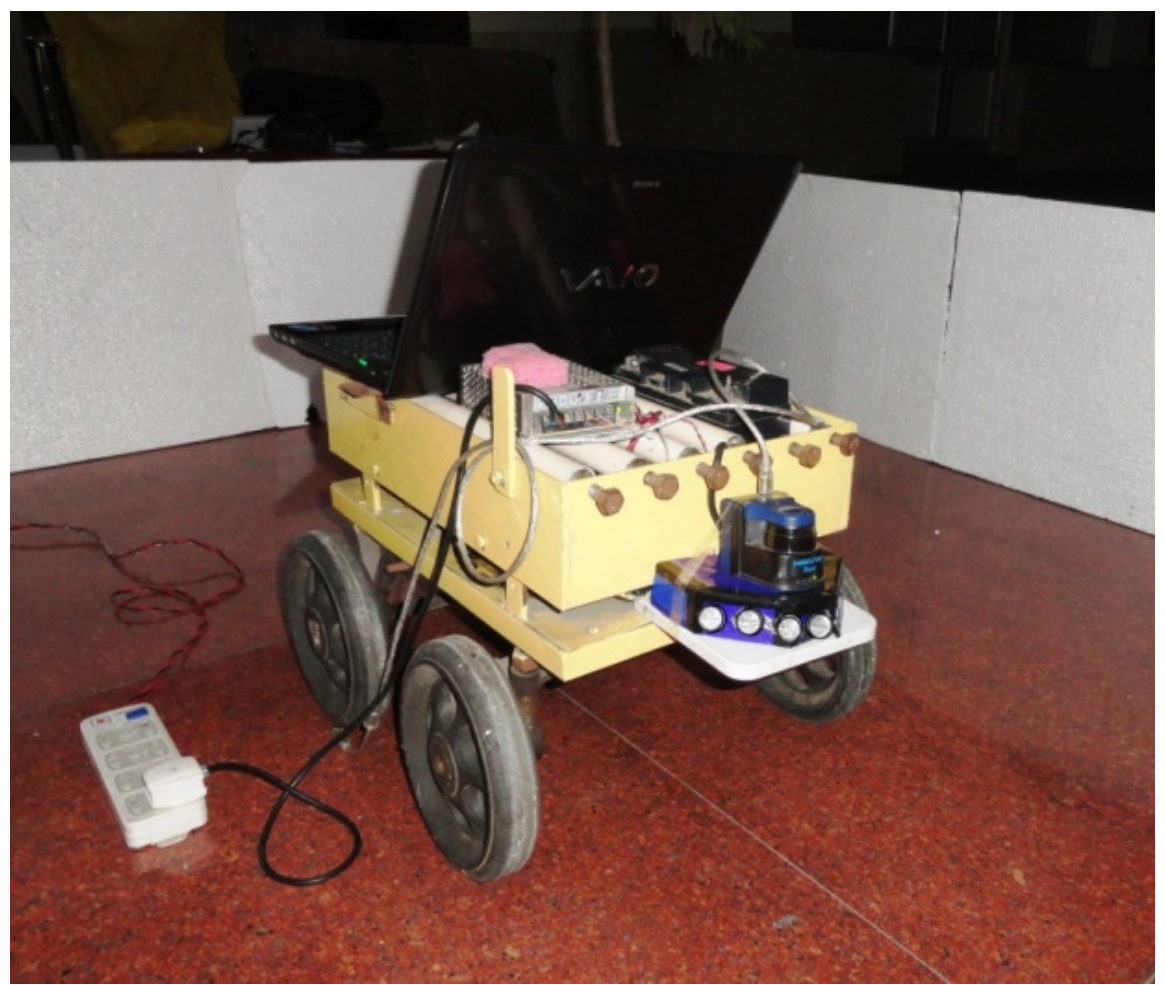

Figure 1. Mobile robot fitted with LRF and Sonar sensors

The \#1 environmental set up is showing various obstacles, robot, and window given in Figure 2 . The robot consists of a ring of seven sonar sensors (LV-Max Sonar-EZ1) and a Laser range finder (Hokuyo UBG04LX-F01), the detail is given in the assumptions. Laser range finder is interfaced to the host computer using USB cable set with maximum transfer rate of 9 Mbps.

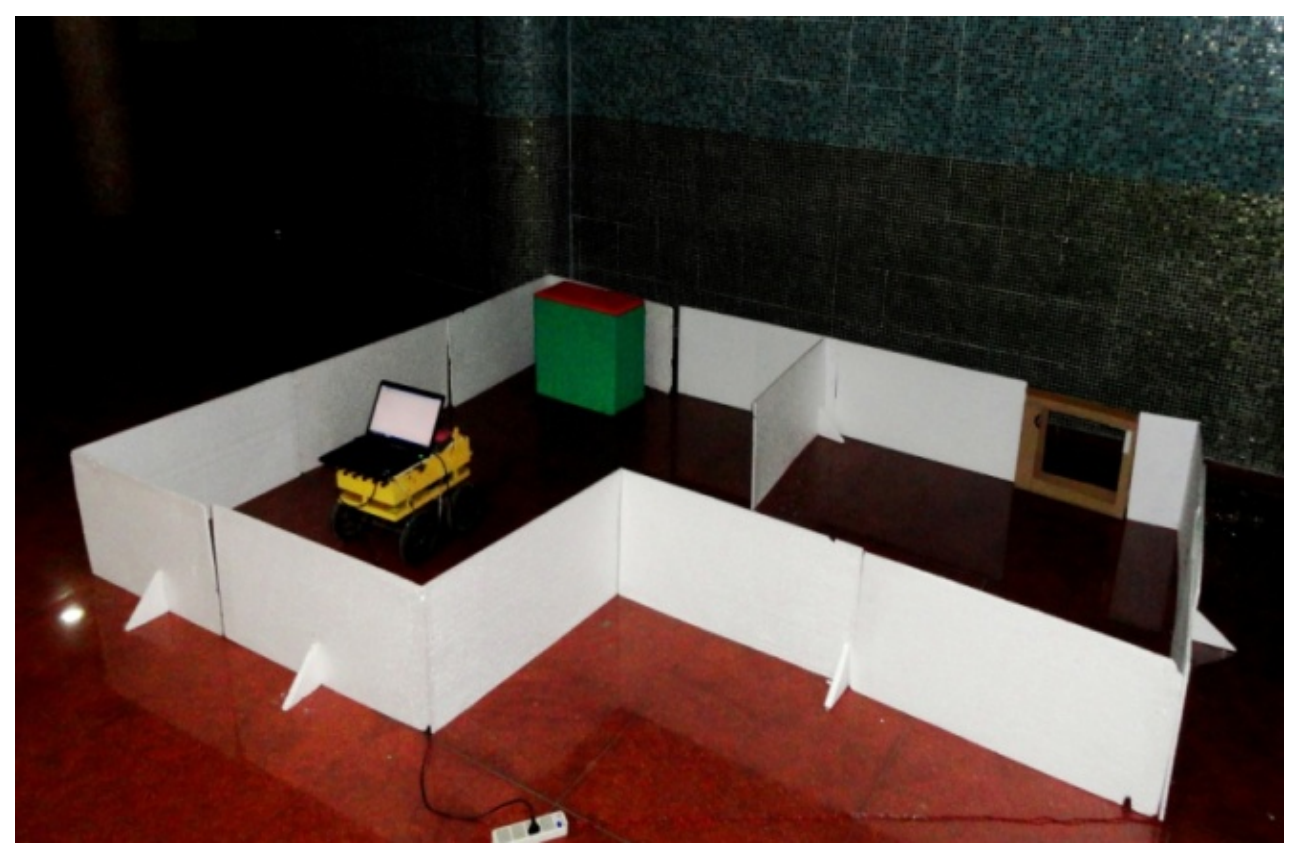

Figure 2. The \#1 environment setup showing mobile robot, obstacles and window 
The sensory information from sonar and laser is transferred to the individual sensor model as per Figure 3 A programmable electronic circuit triggers the sonars asynchronously in anticlockwise direction where the time lag between two successive sonar trigger is set accordingly [14], 1993]. Sonar analog output voltage signal is transferred to data acquisition system through multi-core standard cable. The data is processed on windows 7 professional (64-bit), processor: Intel core i5, $2.53 \mathrm{GHz}$, core 2, threads 4 with simulation code is given in [9]. Microsoft office spreadsheet used to store the sensory information at different locations of the computer memory.

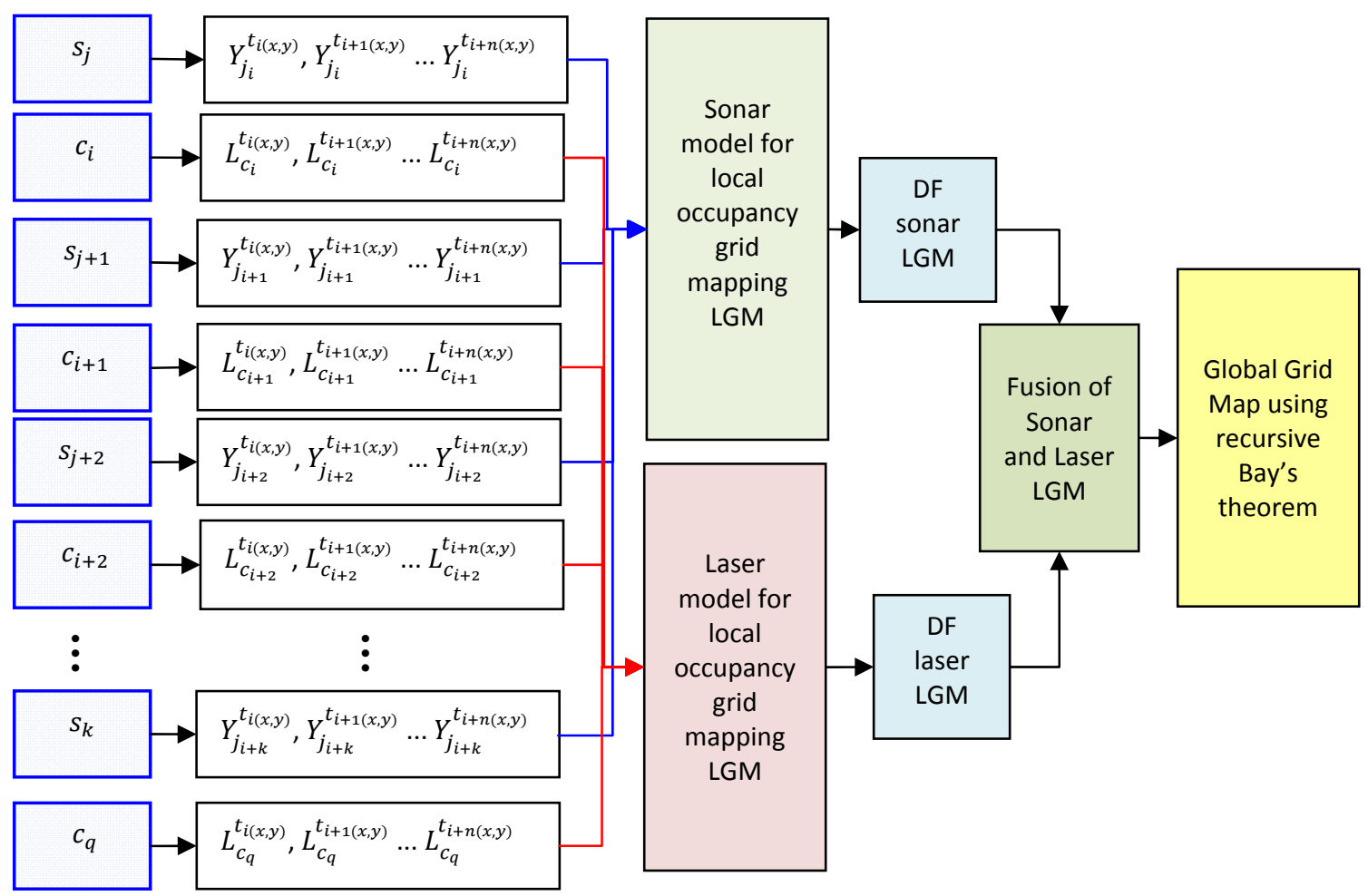

Figure 3 Sensory information flow schematic and sensor fusion frame work with DF

\subsection{Sonar information flow}

The sensory observations for sonar sensors $S_{j}, \ldots, S_{k},($ where $j=1,2, \ldots, k)$ and $k$ is the maximum number of sensors fitted in a ring. At any time step $t$, for mobile robot position coordinates $(x, y)$ is defined as $y_{j}^{t_{i(x, y)}}$, where $i=1,2, \ldots, n$. The next observation of sensor $S_{j}$ at time $(t+1)$ for $(x, y)$ is defined as $y_{j}^{t_{i+1}(x, y)}$. The information flow for next observation is shown in Figure 3. The joint information from set of seven independent sonar sensors is used to construct local grid map (LGM) of dimensions $a_{i} \times b_{i}$.

\subsection{Laser information flow}

The current observation of the laser scanner for group $c_{i}$ at any time step $t$, for mobile robot position coordinates $(x, y)$ defined as $L_{c_{i}}^{t_{i}(x, y)}$, where $i=1,2, \ldots, n$. The next observation of $c_{i+1}$ group at time $(t+1)$ at $(x, y)$ is express as $L_{c_{i+1}}^{t_{i+1}(x, y)}$. The flow of the information for succeeding observation is given in Figure 3. The joint information from different laser group is used to construct local grid map (LGM).

Conclusion Remarks: Hokuyo UBG-04LX-F01 Laser range finder used for study has a range of 20 to 5,600 $\mathrm{mm}$ for $240^{\circ}$, with scan time $28 \mathrm{~m} \mathrm{sec} / \mathrm{scan}$, and angular resolution of $0.36^{\circ}\left(360^{\circ} / 1,024 \mathrm{steps}\right)$. 


\subsection{Sonar and Laser Model for mapping}

Murphy (2004) describes a sensor model as a concept of physical sensing process in which the sensor is extracting relevant data from the environment [33]. The basic model of sonar beam has a field of view specified by $\beta$, the half angle representing the width of the cone, and $R$ is the maximum range it can detect. For grid element from where the range returns the probability of occupancy and probability of emptiness is computed as:

$P($ Occupancy $)=[\{(R-r) / R\}+\{(\beta-\alpha) / \beta\}] / 2 \times$ max. occupied

$P($ Empty $)=1.0-P($ Occ. $)$

where $r$ and $\alpha$ are the distance and angle to the grid element respectively. The term in eq. (2) captures the idea that the closer the grid element to the acoustic axis, the higher is the belief. Likewise, the nearer the grid element is to the sonar beam, the higher the belief (the $\{(R-r) / R\}$ term). The max. occupied term expresses the assumption that a reading of occupied is never fully believable. The term max . occupied $=0.98$ means that, a grid element can never have a probability of being occupied greater than 0.98 . The occupancy of individual cell for the grid element in region between the target object and sensor is calculated using the following:

$P($ Occupied $)=1.0-P($ Empty $)$

$P($ Empty $)=[\{(R-r) / R\}+\{(\beta-\alpha) / \beta\}] / 2$

Successful simulation code for grid evaluation is explained in [9] and similar relation is used to model laser sensor, where the elements of optic axis are consider for grid evaluation. Bayesian is a statically inference method in which observations are used to update or infer the probability that a hypothesis may be true $[4,33]$. The attraction of the Bayesian inference approach is to map building stems from the fact that the Bayes updating rule is recursive [27].

(Bayes' Rule) If the event $B_{1}, B_{2}, \ldots \ldots B_{K}$ constitute a partition of the sample space $\mathrm{M}$, where $\mathrm{p}\left(B_{J} \neq 0\right)$, for $J=1,2,3, \ldots, K$. then for any even $\mathrm{A}$ in $\mathrm{M}$ such that $\mathrm{P}(\mathrm{A}) \neq 0$,

$P\left(B_{i} \mid A\right)=\frac{P\left(B_{i} \cap A\right)}{\sum_{j=1}^{k} P\left(B_{j} \cap A\right)}=\frac{P\left(B_{i}\right) P\left(A \mid B_{i}\right)}{\sum_{j=1}^{k} P\left(B_{j}\right) P\left(A \mid B_{j}\right)}$ for $i=1,2, \ldots, k$

- $\quad B_{i}$ is one of $i$ mutually exclusive (disjoint) event to be estimated.

- $A$ is the evidence event.

- Unconditional probability, $P\left(B_{i}\right)$ is the prior probability of the event $B_{i}$. (called Prior Probabilities).

- The conditional probability $P\left(B_{i} \mid A\right)$ is the probability of $B_{i}$ given $A$. It is also called the posterior probability because it is derived from or dependent upon the specified value of $A$

$P\left(B_{i} \mid A\right)=\frac{P\left(A B_{i}\right)}{P(A)}=\frac{P\left(A \mid B_{i}\right) P\left(B_{i}\right)}{P(A)} \quad$ (Multiplication rule)

Where denominator $P(A)$ is average conditional probability

- $\quad P\left(A \mid B_{i}\right)$ is the conditional probability of $A$ given $B_{i}$

- $\quad \sum_{j=1}^{k} P\left(B_{j} \cap A\right)=\sum_{j=1}^{k} P\left(B_{j}\right) P\left(A \mid B_{j}\right)$ is the total probability of A, and acts as a normalizing factor [4].

Due to the recursive characteristics of bayes rule it is used in mobile robots application for building a map. Bayes' rule is applied to update the occupancy grid for multiple sensor readings $\left(s_{1}, \ldots \ldots, s_{n}\right)$. The $(6)$ transformed to (8) and (9) to the occupancy grid framework for multiple sensor readings.

$P_{i, j}^{o \mid s}=P_{i, j}^{s l o} P_{i, j}^{o} /\left[P_{i, j}^{s l o} P_{i, j}^{o}+\left(1-P_{i, j}^{s \mid o}\right)\left(1-P_{i, j}^{o}\right)\right]$

$P_{i, j}^{e l s}=P_{i, j}^{s l e} P_{i, j}^{e} /\left[P_{i, j}^{s l e} P_{i, j}^{e}+\left(1-P_{i, j}^{s l e}\right)\left(1-P_{i, j}^{e}\right)\right]$ 
The following statements are defined

- In eq. (6) the relevant evidence $A$ is given by sensor reading s as per eq. (8)

- The relevant evidence of true parameter $B_{i}$ is given by $P_{i, j}^{o}$ and $P_{i, j}^{e}$, meaning that they are the prior probabilities of the cell $C_{i, j}$ being occupied or empty they are taken from the existing map.

- The conditional probability $P\left(A i \mid B_{i}\right)$ is given by $P_{i, j}^{s l o}$ and $P_{i, j}^{s l e}$, which are the conditional probabilities that a sensor reading will exit given the state of the cell $C_{i, j}$, being occupied or empty. This conditional probability is given by the probabilistic sensor model.

- The conditional probability $P\left(B_{i} \mid A\right)$ is given by $P_{i, j}^{o \mid s}$ and $P_{i, j}^{e l s}$, which is the conditional probability that a cell is occupied based on the past sensor readings it is the new estimate.

\section{MAP FORMATION}

With reference to $\# 1$ environment the sonar sensor information from sensor $S_{1}, \ldots, S_{7}$, at time step $t$, at position coordinates $(x, y)$ is represented as $y_{1}^{t_{1(72,63)}}$, for sonar sensor $S_{1}$, and $y_{2}^{t_{1(72,63)}}$ for sonar sensor $S_{2}$ and succeeding sensors information from starting point position coordinates $(72,63)$ is stored to the primary data acquisition space and then transferred to sonar sensor model. The sensor model represents the range into occupancy grid from origin $(72,63)$ and stores the grid map information in auxiliary memory space of the processor. The Sonar LGM and Laser LGM are updated by using recursive bayes '(8)' and '(9)'. After series of the observations the complete map of \#1 environment is given in Figure 4. The map in Figure 4 is showing the uncertainty and distortion in the boundaries of the grid map. The Stapen approach eq. (1) is applied to Sonar LGM and Laser LGM before final updating. The experiment is further conducted for \#2, \#3, and \#4 environments.

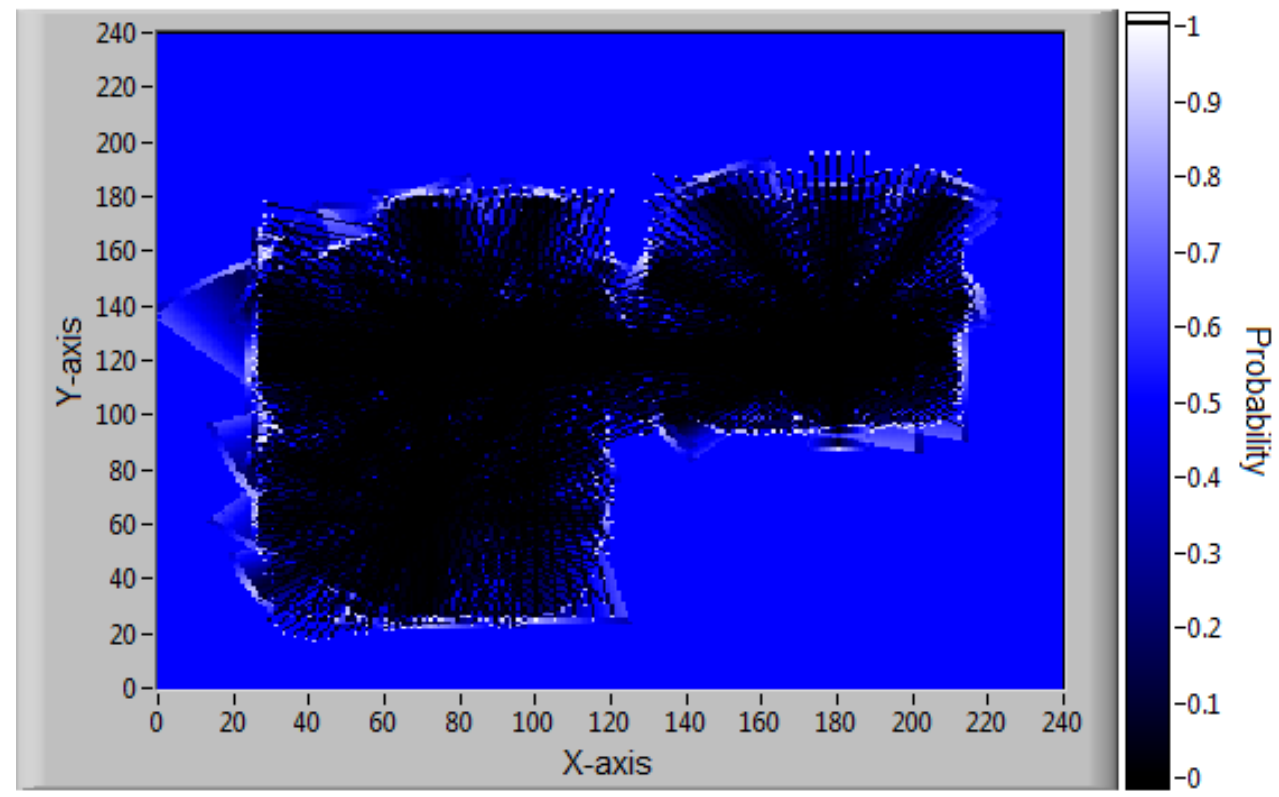

Figure 4. complete map of \#1 environment

\section{QUALITATIVE AND QUANTITATIVE ANALYSIS OF CONVENTIONAL APPROACH}

Figure 4 shows occupancy grid but still there is need to improve further as the boundaries are distorted. The occupancy grid generated by sonar and laser sensor is individually examined. The qualitative analysis is made by comparing the blue print of reference map with generated map. In quantitative analysis random block samples of occupancy gird are selected form different locations of \#1, \#2, \#3, and \#4 environments. The mean probability $\frac{\sum_{i=1}^{n} x_{i}}{n}$ and standard deviation $\frac{\sqrt{\sum(x-\bar{x})^{2}}}{(n-1)}$ of sample is evaluated as given in Table 1. The following observations have been identified, as Table 1.

\subsection{Analysis for sonar grid map}


The qualitative analysis shows that at certain places the boundaries of the map are not matching in respect to the reference map. The quantitative analysis shows that the main cause of distortion is due to the wide angle of sonar, where the average occupancy near the edges of the cone is 0.5-0.66; however the occupancy of the cell at the acoustic axis of sonar is ranging from 0.78 to 0.98 , the maximum and minimum value of data is shown in Table 1 . The average occupancy of cell within the empty area varies from 0-0.26

Table 1. Statistical analysis of occupancy grid

\begin{tabular}{lllllll}
\hline \multirow{2}{*}{ Environment } & & \multicolumn{3}{c}{ Stepan Approach } & \multicolumn{2}{c}{ Proposed approach using DF } \\
& $\begin{array}{l}\text { Sample } \\
\text { (position) }\end{array}$ & Total cells & $\begin{array}{l}\text { Mean } \\
\text { Probability }\end{array}$ & $\begin{array}{l}\text { Standard } \\
\text { Deviation }\end{array}$ & $\begin{array}{l}\text { Mean } \\
\text { Probability }\end{array}$ & $\begin{array}{l}\text { Standard } \\
\text { Deviation }\end{array}$ \\
\hline \multirow{4}{*}{$\# 1$} & P1 & 136 & .65 & .214 & .78 & .168 \\
& P2 & 140 & .08 & .06 & .02 & .044 \\
& P3 & 88 & .57 & .167 & .52 & .114 \\
$\# 2$ & P4 & 110 & .68 & 1.45 & .75 & .182 \\
& G1 & 126 & .72 & .184 & .71 & .142 \\
& G2 & 80 & .62 & .133 & .69 & .174 \\
& G4 & 132 & .57 & .241 & .52 & .196 \\
& H1 & 64 & .02 & .07 & .02 & .04 \\
& H2 & 142 & .67 & .197 & .76 & .146 \\
\hline
\end{tabular}

\subsection{Analysis for Laser grid map}

The map of the laser grid map is not much thicker as sonar grid due to the selection of low angular resolution ${ }^{1}$. The average occupancy of empty area in laser mapping is varying from 0-0.25.

${ }^{1}$ the high angular resolution not considered because it is difficult to handle the huge laser data for mapping. For fusion laser information with sonar information it is necessary to maintain the sensor registry, with the large laser data it is difficult to make ridged compatibility of sensors hence for this experiment limited number of laser beams are selected in one group.

Conclusion Remarks: it is observed that the probability distribution by sonar is different from laser sensor, hence there in a need of special filter for individual maps. It is proposed that the distortion in map may be reduced by implementation of dedicated filter, whose optimal values are selected in respect to the observations of table 1 .

\section{SENSOR FUSION FRAME WORK WITH DEDICATED FILTERS}

To increase in the robustness of map '(10)' and '(11)' is proposed, where the optimal values are selected after qualitative and quantitative analysis is explained in section III. For sonar filter the evaluation criterion for single cell $C_{i, j}$ is given as follows:

$C_{i, j}$ is a single cell in one of the source grid to be evaluated and $\mathrm{P}_{\mathrm{i}, \mathrm{j}}^{\mathrm{o}_{\mathrm{s}}}$ the probability of single cell being occupied based on sonar sensor readings, the probability of individual cell is enhanced as:

$$
P_{i, j}^{o_{s}}=\left\{\begin{array}{cr}
01 & \text { for } P_{i, j}^{o_{s}}>.76 \\
0.8 & \text { for } .52>P_{i, j}^{o_{s}}<0.76 \\
0.5 & \text { for } 0.45>P_{i, j}^{o_{s}}<.54 \\
0 & \text { for } 0>P_{i, j}^{o_{s}}<.26 \\
P_{i, j}^{o_{s}} & \text { otherwise }
\end{array}\right.
$$

The occupancy of the laser grid is enhanced by implementation of eq. (11) where $\mathrm{P}_{\mathrm{i}, \mathrm{j}}^{\mathrm{O}_{\mathrm{L}}}$ the probability of single cell being occupied is based on Laser sensor readings, so that 
$P_{i, j}^{o_{L}}=\left\{\begin{array}{c}1 \\ 0 \\ P_{i, j}^{o_{L}}\end{array}\right.$

$$
\begin{gathered}
\text { for } P_{i, j}^{o_{L}}>.81 \\
\text { for } 0>P_{i, j}^{o_{L}}<.26 \\
\text { otherwise }
\end{gathered}
$$

The filters are implemented to different environment as the results shown in Figure 5 it represents the reference grid map along with results of DF for \#1 environment.

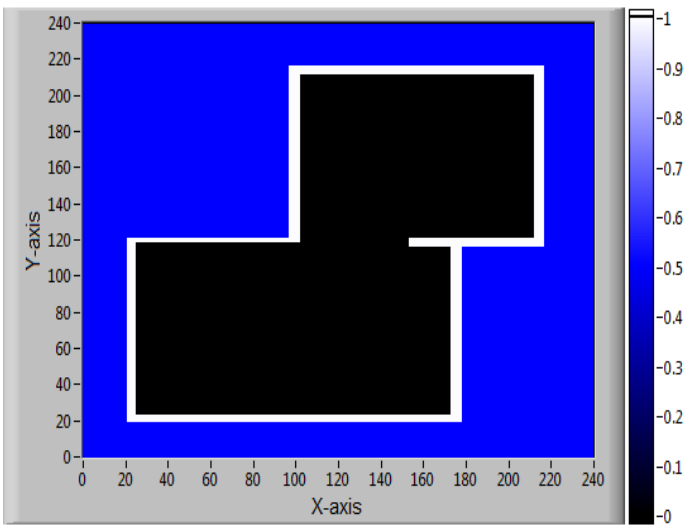

(a)

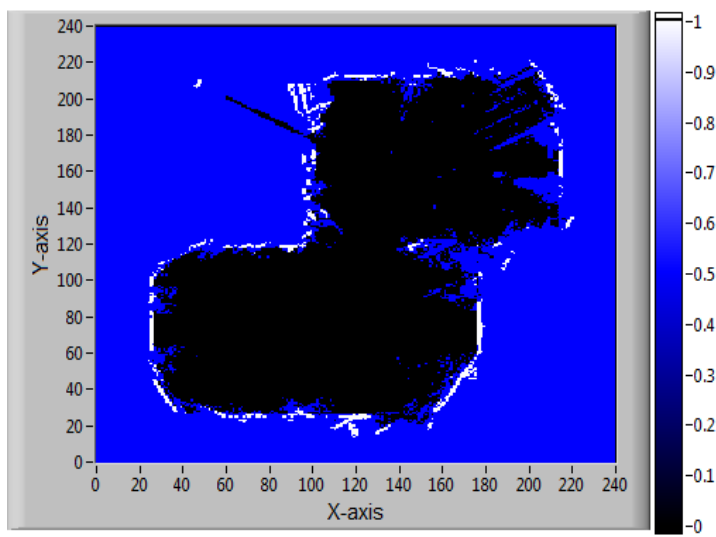

(c)

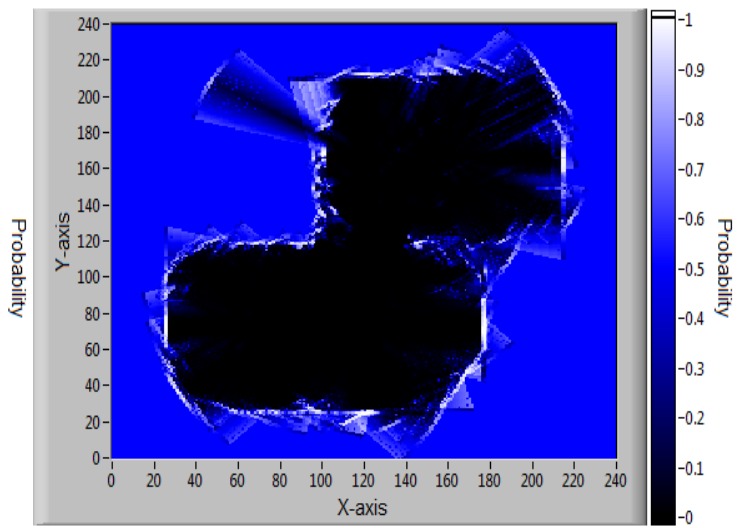

(b)

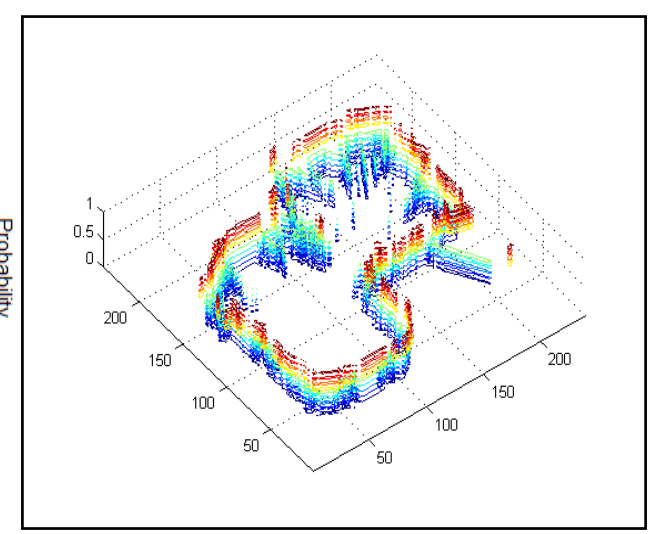

(d)

Figure 5 Mapping of \#1 environment with different methods : (a) reference grid map for \#1 environment (b) map of \#1 environment with conventional approach (c) \#1 map after implementation of dedicated filter (d) $3-\mathrm{D}$ representation of $\# 1$ environment

\section{COMPARISON OF METHOD AND PERFORMANCE ANALYSIS}

The results of mapping with different fusion strategies are compared with newly developed DF approach. The following comparisons are made:

Qualitative comparison: Data of the fused grid map is combined with the blue print of the reference map for different environment. The following observations are made:

a.) Sonar based resultant grid map is qualitatively compared with the reference grid. It is observed that due to the wide beam of sonar sensor the depth of the object is not identified by using sonar sensors. The stapen approach using (1) is applied to the occupancy grid generated by the fusion of sonar and laser as per layer structure the map is improved but still imperfections are persisting in an occupied area near the boundaries Figure 5 (b).

b.) The full comparison is made with the implementation of DF the quality of the map in Figure 5 ( c), show that with the boundaries of the resultant map are very near to the reference map where 3-D map is showing the strong evidence of wall and other obstacles in the environment. 


\section{CONCLUSIONS}

Novel architecture of sensor fusion framework with dedicated filter is proposed to increase the robustness of the occupancy grid for indoor environment. The systematic information flow within the system represents the easy data handling technique for sensor fusion in mobile robots. The updated results using proposed filter shows the improvement in occupancy grid.

Critical investigation of conventional grid shows that the numerical data of sonar based occupancy grid is different from laser grid due to their different modalities and sensor inaccuracies. Several indoor experiments confirmed that the proposed approach is a good solution to improve the quality of the map. It is also noticed that with the implementation of DF, unwanted grid evidence generated due to the diffused sonar information and angular uncertainty is also reduced. Marginal uncertainty on the boundaries are observed i.e. due to wide beam of the sonar where the fused results shows the double boundaries can be consider as the future work. The similar model with can be experimented for outdoor environment and for other sensors such as Radar and IR.

\section{REFERENCES}

[1] Elfes, Using occupancy grids for mobile robot perception and navigation, Computer 22 (6) (1989), 46-57.

[2] Alfredo Chavez Plascencia, Sensor fusion for autonomous mobile robot navigation, Ph.D. Thesis, Department of Electronic System Automation and Control, Aalborg University Denmark, 2004-2007.

[3] FArturo Gil, Oscar Reinoso, Monica Ballesta, Miguel Julia, Multi-robot visual SLAM using a Rao-Blackwellized particle filter, Robotics and Autonomous Systems 58(1) (2010) 68-80.

[4] Boris Lau, Christoph Sprunk, Wolfram Burgard, Efficient grid-based spatial representations for robot navigation in dynamic environments, Robotics and Autonomous Systems, In Press, Accepted Manuscript, Available online 30 August 2012

[5] [5] D. Marinakis, G. Dudek, Pure topological mapping in mobile robotics, IEEE Transactions on Robotics 26(6) (2010) 1051-1064.

[6] D. Rawlinson, R. Jarvis, Ways to tell robots where to Go-directing autonomous robots using topological instructions, IEEE Robotics \& Automation Magazine 15(2) (2008) 27-36.

[7] Dezhen Song, Chang-Young Young Kim, Jingang Yi, Simultaneous localization of multiple unknown and transient radio sources using a mobile robot, IEEE Transactions on Robotics 28(3) (2012) 668-680.

[8] G. Grisetti, C. Stachniss, W. Burgard, Improved techniques for grid mapping with rao-blackwellized particle filters, IEEE Transactions on Robotics 23(1) (2007) 34-46.

[9] Gaurav Sharma, K.S. Nagla, Evaluation of occupancy grid using LabVIEW software, ACODS: International conference on Advances in Control and Optimization of Dynamical Systems, IISc Bangalore, February 13, 2012

[10] H. Durrant-Whyte, T. Bailey, Simultaneous localization and mapping: part-I, IEEE Robotics \& Automation Magazine 13(2) (2006) 99-110.

[11] Ian Lane Davis, Anthony Stentz, Sensor fusion for autonomous outdoor navigation using neural networks, IEEE International Conference Intelligent robots and systems 3 (1995) 338-343.

[12] J.J. Leonard, H.F. Durrant-Whyte, Mobile robot localization by tracking geometric beacons, Robotics and Automation 7(3) (1991) 376-382.

[13] J.J. Leonard, H.F. Durrant-Whyte, Simultaneous map building and localization for an autonomous robot, in: IEEE/RSJ International Workshop on Intelligent Robots and Systems, 1991, pp. 1442-1447.

[14] Johann Borenstein, Yoram Koren, Error-eliminating rapid ultrasonic firing, US Patent, 5239515, 1993

[15] Jose-Luis Blanco, Javier Gonzalez, Juan-Antonio Fernandez-Madrigal, A new method for robust and efficient occupancy grid-map matching, 3rd Iberian Conference on Pattern Recognition and Image Analysis, 6-8 June 2007

[16] K.S. Nagla, MoinUddin, Dilbag Singh, Nikhil Rathi, Sensor fusion frame work for marble/stone processing industry using low cost sensors, 22nd DAAAM World Symposium Intelligent Manufacturing \& Automation: Power of Knowledge and Creativity, at Austria Center Vienna, Vienna, Austria, 23-26th November 2011, pp 339340.

[17] K.S. Nagla, MoinUddin, Dilbag Singh, Rajeev Kumar, Object identification in dynamic environment using sensor fusion, Applied Imagery Pattern Recognition (AIPR) annual workshops, USA, 2010.

[18] L. Conde Bento, Urbano Nunes, Fernando Moita, Antonio Surrecio, Sensor fusion for precise autonomous vehicle navigation in outdoor semi-structured environment, Proceedings of the 8th International IEEE Conference on Intelligent Transportation Systems Vienna, Austria, 2005, pp 245-250.

[19] L. Jetto, S. Longhi1, D. Vitali, Localization of a wheeled mobile robot by sensor data fusion based on a fuzzy logic adapted kalman filter, Control Engineering Practice 7 (1999) 763-771.

[20] M. Asada, Y. Fukui, S. Tsuji, Representing global world of a mobile robot with relational local maps, IEEE transaction Systems, Man and Cybernetics 20(6) (1990) 1456-1461.

[21] M. Khatib, T. Simeon, Sensor-based motion planning and control for the HILARE mobile robot, International Conference on Intelligent Robots and Systems, 1997, pp. V8-V9.

IJRA Vol. 4, No. 1, March 2015:82-92 
[22] M. Mucientes, D.L. Moreno, A. Bugarn, S. Barro, Design of a fuzzy controller in mobile robotics using genetic algorithms, Applied Soft Computing 7 (2007), 540-546.

[23] Mohammad Reza Asharif, Behzad Moshiri, Reza HoseinNezhad, Sensor fusion by Pseudo Information Measure: A Mobile Robot Application, ISA Transactions 41 (2002) 283-301.

[24] Mostofi, Yasamin, Cooperative wireless-based obstacle/object mapping and see-through capabilities in robotic networks, IEEE Transactions on Mobile Computing 12(5) (2013) 817-829.

[25] N. Ghosha, Y.B. Ravi, A. Patra, S. Mukhopadhyay, S. Paul, A.R. Mohanty, A.B. Chattopadhyay, Estimation of tool wear during CNC milling using neural network-based sensor fusion, Mechanical Systems and Signal Processing 21 (2007) 466-479.

[26] Naoki Suganuma, Toshiki Matsui, Robust environment perception based on occupancy grid maps for autonomous vehicle, IEEE, SICE Annual Conference, 2010, pp. 2354-2357.

[27] Nicolas Viandier, Juliette Marais, Asma Rabaoui, Emmanuel Duflos, Studies on DPM for the density estimation of pseudorange noises and evaluations on real data, IEEE proceedings on Position Location and Navigation Symposium (PLANS), 2010, pp. 1154-1161.

[28] Ofir Cohen, Yael Edan, A sensor fusion framework for online sensor and algorithm selection, Robotics and Autonomous Systems, (56), (2008) pp 762-776.

[29] [29] Petr Stepan, Miroslav Kulich, Libor Preucil, Robust data fusion with occupancy grid, IEEE Transactions on Systems, Man, and Cybernetics — part c: applications and reviews 35 (2005) 106-115.

[30] R. Talluri, E.M. Nebot, H. Durrant-Whyte, An evidential approach to map-building for autonomous vehicles, IEEE Transactions on Robotics and Automation 14(4) (1998) 623-629.

[31] R. Talluri, J.K. Aggarwal, Image map correspondence for mobile robot self-location using computer graphics, Pattern Analysis and Machine Intelligence 15(6) (1993) 597-601.

[32] R.C. Luo, C.C. Lai, Enriched indoor map construction based on multisensor fusion approach for intelligent service robot, IEEE Transactions on Industrial Electronics 59(8) (2012) 3135-3145.

[33] Robin R. Murphy, AI in Robotics, Prentice Hall of India private limited, New Delhi, 2004

[34] Sebastian Thrun, Learning occupancy grid maps with forward sensor models, Autonomous Robots 15 (2003) 111127.

[35] SungHwan Ahn, Jinwoo Choi, NakFu Lett Doh, Wan Kyun Chung, A practical approach for EKF-SLAM in an indoor environment: fusing ultrasonic sensors and stereo camera, Autonomous Robot 24 (2008) 315-335.

[36] Sv. Noykov, Ch. Roumenin, Occupancy grids building by sonar and mobile robot, Robotics and Autonomous Systems 55 (2007) 162-175.

[37] Thien-Nghia N. Nguyen, Bernd Michaelis, Ayoub K. Al-Hamadi, Michael Tornow, Marc-Michael Meinecke, Stereo-Camera-Based Urban Environment Perception Using Occupancy Grid and Object Tracking, IEEE Transactions on Intelligent Transportation Systems 13(1) (2012) 154-165.

\section{BIOGRAPHIES OF AUTHORS}
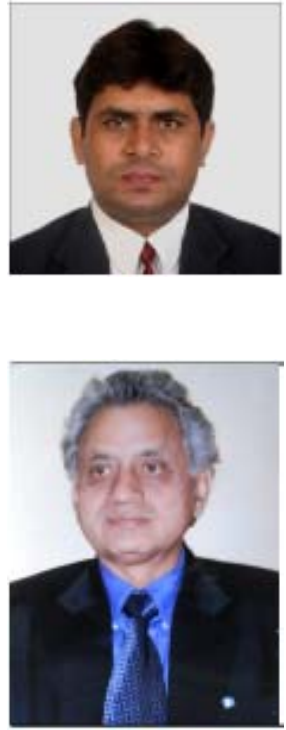

KS Nagla is Ph.D. research scholar in the Department of Instrumentation and Control Engineering, at Dr. B.R. Ambedkar National Institute of Technology Jalandhar. He has completed his B.Tech. (Degree) in electronic and instrumentation from Punjabi University Patiala and his M.Tech in instrumentation and control engineering (specialization robotics) from Dr. B.R. Ambedkar National Institute of Technology Jalandhar. He, with his team, has developed several robots and new mechanisms during the past ten years. He is an inventor of three inventions (Indian patents) and many more inventions are in the process of being granted. His current area or research is articial intelligence in mobile robots and industrial automation.

Dr. Moin Uddin completed his Ph.D. from the Indian Institute of Technology (IIT) Roorkee, India in year 1993. His area of research is robotics, computer networking, AI and Soft Computing. He is a member of various national and international technical professional bodies/societies. He is recipient of the Dr. Radha Krishnan Memorial Award-96. At present he is working as Pro-Vice-Chancellor at Delhi Technological University Delhi-India. 


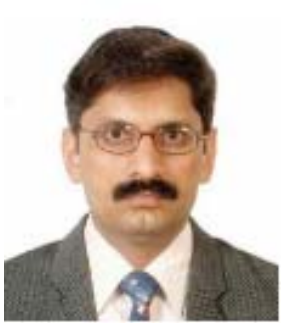

Dr. Dilbag Singh received the B.E. (Hons.) degree in electrical engineering from Punjab Engineering College, Chandigarh in 1991, and the M.E. degree in control and guidance from the University of Roorkee in 1993, and the Ph.D. degree in engineering from the Indian Institute of Technology Roorkee, in 2004. He is presently serving as Associate Professor of Instrumentation and Control Engineering at Dr. B.R. Ambedkar National Institute of Technology Jalandhar. His research interests are in signal processing, sensors and biomedical applications. 